\title{
Quantitative genetic analysis of courtship and reproduction in female Drosophila melanogaster
}

\author{
Mark H. Gromko
}

Department of Biological Sciences, Bowling Green State University, Bowling Green, Ohio 43403, U.S.A.

Three hundred mother-daughter pairs were analyzed for seven attributes related to courtship and reproduction. Only the lag time from first courtship to copulation was significantly heritable; genetic correlations involving this attribute were not significant. The genetic correlation between fertility and lag time to first courtship was negative and significant. However, this genetic correlation is expected to have little impact on the retention of additive genetic variance or on response to selection because it involves two attributes with low heritabilities. The pattern of phenotypic covariation among traits is largely explained by environmental causes and is consistent with that found in a previous analysis of father-son pairs (Gromko, 1987).

\section{INTRODUCTION}

Simultaneous selection for two characters is expected to fix alleles that affect both characters favourably and eliminate alleles that affect both characters unfavourably. Genetic variation which remains will involve alleles that have opposite effects on the two characters, thus producing a negative genetic correlation due to antagonistic pleiotropy (Dickerson, 1955; Robertson, 1955; Falconer, 1981, p. 300; Lande, 1982; Rose, 1982). Such antagonistic pleiotropy might cause the retention of additive genetic variation for each of the two traits; whereas in the absence of such pleiotropic effects selection is expected to deplete the additive genetic variance for both. By the same logic, the existence of a negative correlation between two traits which each have nonzero heritabilities might be taken as evidence of past natural selection on those two traits (Falconer, 1981).

The existence of negative genetic correlation between heritable traits has been demonstrated in a number of organisms (Rose and Charlesworth, $1981 a, b$; Berenbaum et al., 1986; Palmer and Dingle, 1986; Gromko, 1987; Arnold, 1988). In one case (Gromko, 1987), seven attributes of courtship and reproduction were measured in 330 father-son pairs of Drosophila melanogaster. Copulation duration had significant heritability; there was a large negative genetic correlation between copulation duration and courtship vigour, and a positive genetic correlation between copulation duration and fertility. However, in order to use such information to predict selection response, data on females must also be gathered, since all the aspects of courtship and reproduction measured are not "male" or "female" characteristics, but result from the interaction of the two sexes. Thus, I report here a mother-daughter analysis for the same seven attributes.

\section{MATERIALS AND METHODS}

The flies used in this experiment were from the same laboratory population, the JEFFERS population, in the male-focused study (Gromko, 1987). The stock was maintained since it was collected in August 1984 under conditions of minimal inbreeding among 40 lines cultured in 8 -dram vials. For further description of this population and rearing scheme, see Fukui and Gromko (in press).

Virgin males and females were collected every other week from stock minimal-inbreeding cultures and were allowed, at three to five days of age, to mate at random in a small population cage. Virgin males and females to be tested were collected from these one-generation population cage cultures, and were thus random-bred for one generation before 
testing. All flies were maintained at $25^{\circ} \mathrm{C}$ and were on a 12:12 light cycle throughout the culturing and test period.

The procedures used are as similar as possible to those described by Gromko (1987), with the difference that females, not males, were the focal individuals. The major consequence of the change in focal individual is that heritabilities and genetic correlations will be based on additive genetic variance and covariance in females. The change in focal individual will have less of an effect on the pattern of phenotypic covariation. A summary of the experimental design is provided in fig. 1; for a complete description of procedures see Gromko (1987).

The variables LAGCOURT, LAGCOP and 2ND LAGCOURT were normalized by transformation to natural logs. The arcsine transformation was satisfactory for the variable VIGOR. For the variable 2ND VIGOR it was necessary to first transform to arcsines and then to take the natural log. The variables COPDUR and FERTILITY were normally distributed without transformation.

Heritabilities were estimated from the offspring-parent regression and genetic correlations from covariances between offspring and parent. The formulas used for calculating

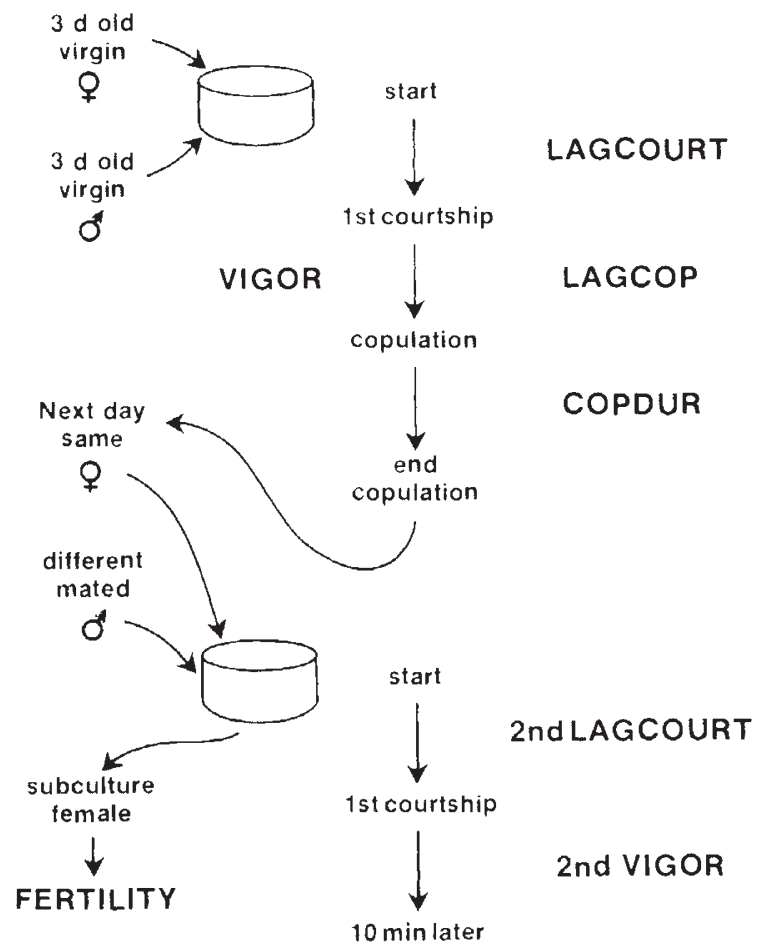

Figure 1 Summary of experimental design. heritabilities, genetic correlations, and their standard errors are the same as given by Gromko (1987). Principal component analysis (with varimax rotation) was used to describe the pattern of phenotypic and environmental variation among females. All statistical analysis relied on SAS (SAS Institute Inc., $1985 a, b$ ).

The experiment was carried out twice, once each by two different technicians. There were small but significant differences between the means of attributes measured in the two separate experiments, so the data were not pooled, but analyzed separately. The pattern of phenotypic, genetic and environmental variation and covariation was very similar in the two replicates. The complete results of the second, and larger, analysis $(N=300$ mother-daughter pairs) will be presented here. The major features of the first $(N=230)$ run will be only briefly mentioned as they were much the same.

\section{RESULTS}

Means and 95 per cent confidence intervals of the seven attributes measured, backtransformed to the original scale of measurement, are shown in table 1 . The differences between mothers' and daughters' means are significant $(t$-test $)$ for LAGCOP $(P<$ $0.0001)$, VIGOR $(P=0.011)$, COPDUR $(P<$ $0.0001)$ and 2 ND VIGOR $(P=0.001)$, but there is no obvious explanation for these differences.

Table 1 Means and 95 per cent confidence intervals calculated on transformed data and back-transformed to the original scale of measurement

\begin{tabular}{|c|c|c|c|}
\hline Variable & $L_{1}$ & Mean & $L_{2}$ \\
\hline \multicolumn{4}{|c|}{ LAGCOURT $(\min )$} \\
\hline mothers & $1 \cdot 08$ & $1 \cdot 21$ & $1 \cdot 34$ \\
\hline daughters & $1 \cdot 18$ & $1 \cdot 31$ & 1.46 \\
\hline \multicolumn{4}{|c|}{ VIGOR (per cent) } \\
\hline mothers & $82 \cdot 2$ & $85 \cdot 1$ & $87 \cdot 8$ \\
\hline daughters & $76 \cdot 5$ & $79 \cdot 6$ & $82 \cdot 5$ \\
\hline \multicolumn{4}{|c|}{$\operatorname{LAGCOP}(\mathrm{min})$} \\
\hline mothers & $3 \cdot 24$ & $3 \cdot 71$ & $4 \cdot 25$ \\
\hline daughters & $5 \cdot 53$ & $6 \cdot 29$ & $7 \cdot 15$ \\
\hline \multicolumn{4}{|c|}{ 2ND LAGCOURT $(\mathrm{min})$} \\
\hline mothers & $1 \cdot 26$ & $1 \cdot 42$ & 1.60 \\
\hline daughters & 1.42 & 1.58 & $1 \cdot 76$ \\
\hline \multicolumn{4}{|c|}{ 2ND VIGOR (per cent) } \\
\hline mothers & $8 \cdot 4$ & $9 \cdot 8$ & $11 \cdot 4$ \\
\hline daughters & $12 \cdot 0$ & $13 \cdot 8$ & $16 \cdot 0$ \\
\hline \multicolumn{4}{|c|}{ COPDUR $(\min )$} \\
\hline mothers & $16 \cdot 8$ & $17 \cdot 3$ & $17 \cdot 7$ \\
\hline daughters & $18 \cdot 4$ & $18 \cdot 9$ & $19 \cdot 4$ \\
\hline \multicolumn{4}{|c|}{ FERTILITY (count) } \\
\hline mothers & $137 \cdot 7$ & $145 \cdot 1$ & $152 \cdot 6$ \\
\hline daughters & $141 \cdot 1$ & $151 \cdot 2$ & $161 \cdot 3$ \\
\hline
\end{tabular}


Table 2 Matrix of phenotypic correlations $\left(r_{\mathrm{p}}\right)$ among mothers' characters

\begin{tabular}{|c|c|c|c|c|c|c|}
\hline $\begin{array}{l}\text { LAGCOURT } \\
\text { VIGOR } \\
\text { LAGCOP } \\
\text { 2ND LAGCOURT } \\
\text { 2ND VIGOR } \\
\text { COPDUR }\end{array}$ & -0.08 & $\begin{array}{l}-0.05 \\
-0.45^{* * *}\end{array}$ & $\begin{array}{r}0.08 \\
-0.04 \\
-0.03\end{array}$ & $\begin{array}{l}-0.04 \\
0.09 \\
-0.04 \\
-0.41^{* * *}\end{array}$ & $\begin{array}{r}0.07 \\
-0.04 \\
0.00 \\
-0.03 \\
0.02\end{array}$ & $\begin{array}{r}-0.01 \\
-0.02 \\
-0.04 \\
0.01 \\
-0.04 \\
0.02\end{array}$ \\
\hline
\end{tabular}

There were significant differences between the temperature $\left(0.25^{\circ} \mathrm{C}\right)$ and relative humidity $(4$ per cent) at which mothers' and daughters' tests were run, and it was thought that these might be responsible for the differences in mothers' and daughters' attributes. However, an analysis of covariance, with temperature and humidity as covariates, still showed significant differences between mothers' and daughters' mean scores. There were also small unexplained differences between mothers' and daughters' scores in the smaller first run of the experiment, although in that run the differences were found for LAGCOURT, VIGOR, COPDUR, and 2ND LAGCOURT. The only bearing these differences have on the results is that they could represent variance due to environmental sources, and such variance would tend to lower the estimates of heritabilities.

There are two significant phenotypic correlations among the seven attributes measured in mothers (table 2). Principal component analysis of this matrix (with varimax rotation) produced three components with eigenvalues greater than 1.0 which together explain 57 per cent of the variation in the data set (table 3 ). A similar analysis done on daughters produced three components with the same pattern of loading. Furthermore, the factor pattern is essentially the same as was pre-
Table 3 Principal component analysis (with varimax rotation) of mothers' phenotypic correlations

\begin{tabular}{lrrr}
\hline & \multicolumn{1}{c}{ PC1 } & \multicolumn{1}{c}{ PC2 } & \multicolumn{1}{c}{ PC3 } \\
\hline LAGCOURT & -0.02 & 0.08 & 0.71 \\
VIGOR & -0.83 & -0.08 & -0.11 \\
LAGCOP & 0.85 & -0.03 & -0.14 \\
2ND LAGCOURT & -0.03 & 0.83 & 0.04 \\
2ND VIGOR & -0.08 & -0.82 & -0.03 \\
COPDUR & 0.15 & -0.15 & 0.67 \\
FERTILITY & -0.08 & 0.09 & 0.34 \\
\hline
\end{tabular}

viously found for males (Gromko, 1987): PC1 represents courtship of virgin flies, and $\mathrm{PC} 2$ represents courtship of mated flies. In the analysis of males (Gromko, 1987) LAGCOURT loaded fairly evenly on all three factors (with or without rotation) whereas here it loads on PC3.

Heritabilities and genetic correlations are given in table 4. The standard error of the heritability estimates is $0 \cdot 115$. The heritability of LAGCOP (more than three times its standard error) is the only significant one in the table. Similarly, there is only one clearly significant genetic correlation, a negative one between LAGCOURT and FERTILITY. Estimates of genetic correlations between traits with small heritabilities frequently fell outside the bounds of $-1 \cdot 0$ to $1 \cdot 0$, as has been found

Table 4 Heritabilities (on diagonal) and genetic correlations ( $r_{\mathrm{A}}$, above diagonal). The S.E.s of genetic correlations are given in parenthesis. No value is reported for any genetic correlation which was smaller than its standard error.

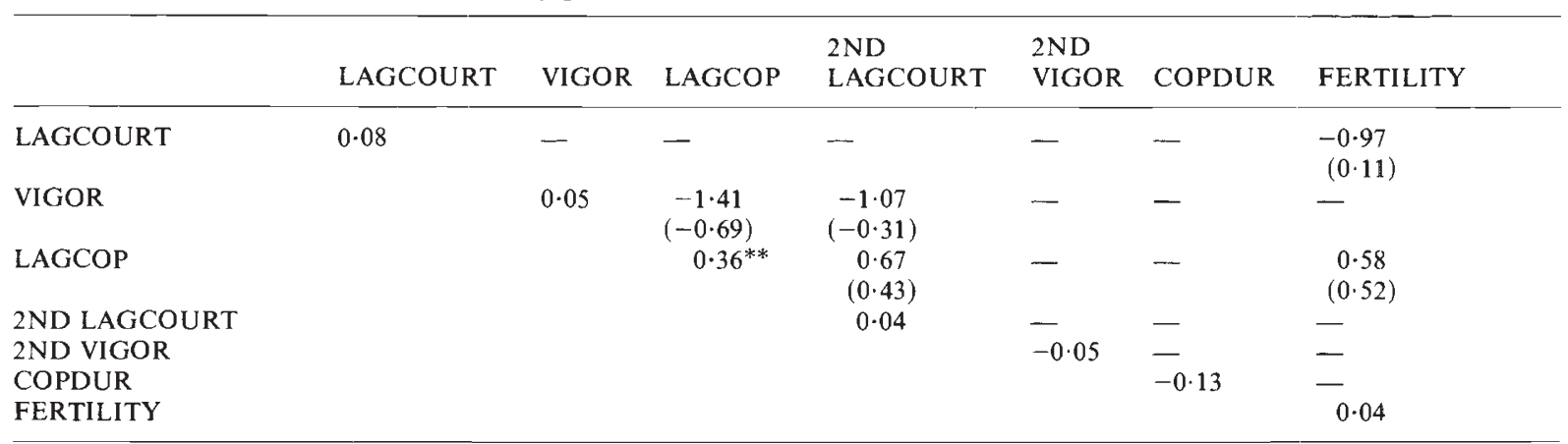


Table 5 Environmental correlations $\left(r_{\mathrm{E}}\right)$

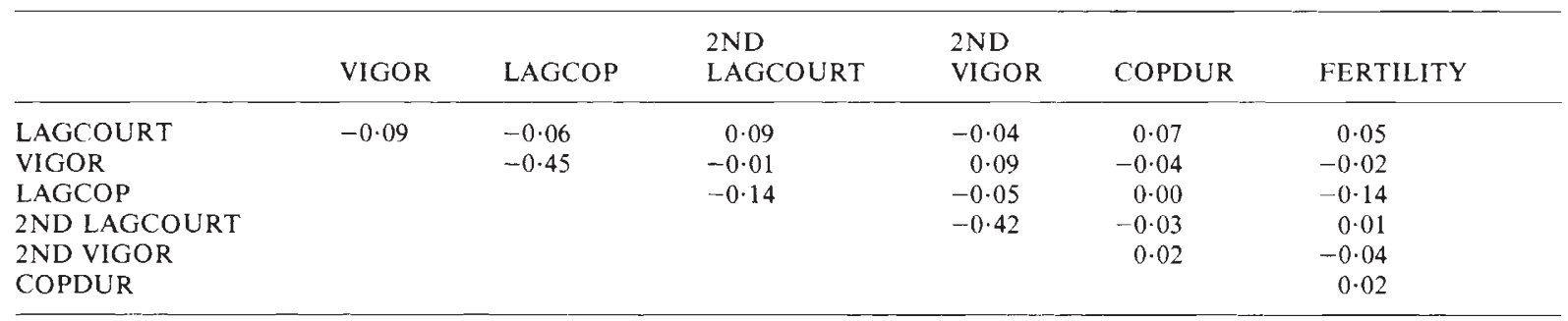

in similar studies (Mitchell-Olds, 1986; Gromko, 1987), and standard errors for these estimates are negative. These estimates are of unknown reliability and significance. Estimates of genetic correlations which were smaller than their standard error are reported as a dashed line, as these undoubtedly estimate a value of zero.

The major features of the genotypic matrix were also evident in the smaller first replicate. The heritability of LAGCOP was significant and of similar magnitude $(0 \cdot 31)$. The genetic correlation between LAGCOURT and FERTILITY, although somewhat smaller $(-0.72)$, was still significant $(\mathrm{S} . \mathrm{E} .=0 \cdot 29)$. The two attributes with negative estimates of heritability in table 4 were positive, but close to zero, in the first replicate, while the heritability of VIGOR in the first replicate was negative.

In order to estimate environmental correlations, the negative estimates of heritability were set to zero, and the genetic correlations that fell outside the bounds of -1.0 and 1.0 were raised or lowered by one S.E. With just one significant genetic correlation and six of seven heritabilities close to zero, it is not surprising that the matrix of environmental correlations (table 5) and the principal components analysis of that matrix (table 6) are very similar to the phenotypic relationships.

Table 6 Principal component analysis (with varimax rotation) on the matrix of environmental correlations

\begin{tabular}{lrrr}
\hline & \multicolumn{1}{c}{ PC1 } & \multicolumn{1}{c}{ PC2 } & \multicolumn{1}{c}{ PC3 } \\
\hline LAGCOURT & 0.06 & 0.14 & 0.67 \\
VIGOR & -0.83 & -0.09 & -0.16 \\
LAGCOP & 0.85 & -0.08 & -0.18 \\
2ND LAGCOURT & -0.12 & 0.84 & 0.04 \\
2ND VIGOR & -0.13 & -0.83 & 0.01 \\
COPDUR & 0.10 & -0.16 & 0.59 \\
FERTILITY & -0.17 & 0.04 & 0.52 \\
\hline
\end{tabular}

\section{DISCUSSION}

Very little of the variation and covariation in the seven attributes of courtship and reproduction measured has a genetic basis in females (table 4). It is noteworthy that the one significant genetic correlation (between LAGCOURT and FERTILITY) does not involve the one attribute which has a significant heritability (LAGCOP). The low heritabilities of LAGCOURT and FERTILITY render the negative genetic correlation between them of little significance. Despite its magnitude, this negative genetic correlation: (1) is not involved in the retention of additive genetic variance (Rose, 1982); (2) would not be expected to produce constraint on selection or indirect response; and (3) has little impact on the pattern of phenotypic covariation (tables 2 and 3 ). We have also carried out a pilot mother-son study of the same seven attributes, and found no evidence of any additive genetic basis between the sexes for variation in these attributes.

The pattern of phenotypic covariation (in both this and the male study, Gromko, 1987) is dominated by the negative correlation between VIGOR and LAGCOP (apparent in PC1) and by the negative correlation between 2ND VIGOR and 2ND LAGCOURT (apparent in PC2). These correlations seem to be attributable to environmental causes (tables 5 and 6), although in this design "environment" must be construed to include effects due to differences among males as well as all environmental and non-additive genetic effects. The correlation between VIGOR and LAGCOP shows that copulations occur more quickly the higher the level of courtship, and this argues for the efficacy of courtship or courtship summation (Cobb et al., 1987). The negative correlation between 2ND VIGOR and 2ND LAGCOURT shows that the more quickly the courtship of a mated female is begun the more vigorous the courtship which follows, which does not appear to be true of virgin flies.

The experimental design is expected to produce the same pattern of phenotypic covariation among the five virgin attributes and between the two mated attributes in the male- and female-focused studies. However, any phenotypic covariation between 
mated and virgin attributes might be different in the male- and female-focused studies. In both this and in the male-focused study (Gromko, 1987), the virgin and mated aspects of courtship loaded on different principal components. The varimax rotation is rigid and produces orthogonal components. Thus, in both males and females, the attributes of virgin courtship and mated courtship that were measured appear to be relatively independent of one another.

The results of this study can be used in conjunction with those of the male-focused study (Gromko, 1987) to predict response to selection. If one were to base selection on the behavior of pairs of males and females, the arithmetic average of the male and female heritabilities would be appropriate. For COPDUR and LAGCOP these values are $h^{2}=0 \cdot 23$ and $h^{2}=0 \cdot 21$, respectively, and would be compared to realized heritability of the selection response. Correlated responses to selection could also be predicted by use of equation 19.6 of Falconer (1981). If ten of 75 pairs were selected $(i=1 \cdot 587)$, and using arithmetic averages of the male and female genetic correlations, selection on COPDUR is predicted to produce a correlated response in VIGOR of about 1.6 per cent per generation, or, if up and down lines were compared, a divergence of about $3 \cdot 2$ per cent per generation. Similarly, FERTILITY would be expected to respond to selection for COPDUR at a rate of about two progeny per generation, or a divergence of four progeny per generation in up and down lines.

Acknowledgements I thank K. Carr and A. Briot, the individuals who conducted the behavioural assays. This work was supported by NSF Grant BNS-8418934.

\section{REFERENCES}

ARNOLD, S. J. 1987. Genetic correlation and the evolution of physiology. In Feder, M. E., Bennett, A. F., Burggren, W. W., and Huey, R. B. (eds) New Directions in Ecological Physiology, Cambridge University Press, Cambridge, pp. 184-215.

BERENBAUM, M. R., ZANGERL, A. R. AND NITAO, J. K. 1986. Constraints on chemical coevolution: wild parsnips and the parsnip webworm. Evolution, 40, 1215-1228.

COBB, M., CONNOLLY, K. AND BURNETT, B. 1987. The relationship between locomotor activity and courtship in the melanogaster species sub-group of Drosophila. Anim. Behav., 35, 705-713.

DICKERSON, G. E. 1955. Genetic slippage in response to selection for multiple objectives. CSHSQB, 20, 213-224.

FALCONER, D. S. 1981. Introduction to Quantitative Genetics, 2nd edn. Longman, London.

FUKUI, H. H. AND GROMKO, M. H. Female receptivity to remating and early fecundity in Drosophila melanogaster. Evolution, (in press).

GROMKO, M. H. 1987. Genetic constraint on the evolution of courtship behaviour in Drosophila melanogaster. Heredity, $58,435-441$.

LANDE, R. 1982. A quantitative genetic theory of life history evolution. Ecology, 63, 607-615.

MITCHELL-OLDS, T. 1986. Quantitative genetics of survival and growth in Impatiens capensis. Evolution, 40, 107-116.

PALMER, J. P. AND DINGLE, H. 1986. Direct and correlated responses to selection among life-history traits in milkweed bugs (Oncopeltus fasciatus). Evolution, 40, 767-777.

ROBERTSON, A. 1955. Selection in animals: Synthesis. CSHSQB, 20, 225-229.

ROSE, M. R. 1982. Antagonistic pleiotropy, dominance, and genetic variation. Heredity, 48, 63-78.

ROSE, M. R. AND CHARLESWORTH, B. $1981 a$. Genetics of life history in Drosophila melanogaster. I. Sib analysis of adult females. Genetics, 97, 173-186.

ROSE, M. R. AND CHARLESWORTH, B. $1981 b$. Genetics of life history in Drosophila melanogaster. II. Exploratory selection experiments. Genetics, 97, 187-196.

SAS INSTITUTE INC. 1985. SAS User's Guide: Statistics, Version 5 Edition, SAS Institute Inc., Cary, NC.

SAS INSTITUTE INC. 1985. SAS User's Guide: Basics, Version 5 Edition, SAS Institute Inc., Cary, NC. 